\title{
Optical activity of neutrinos and antineutrinos
}

\author{
Ali Abbasabadi \\ Department of Physical Sciences, Ferris State University, Big Rapids, Michigan 4930\%, USA \\ Wayne W. Repko \\ Department of Physics and Astronomy, Michigan State University, East Lansing, Michigan 48824, USA
}

(Dated: November 2, 2018)

\begin{abstract}
Using the one-loop helicity amplitudes for low-energy $\nu \gamma \rightarrow \nu \gamma$ and $\bar{\nu} \gamma \rightarrow \bar{\nu} \gamma$ scattering in the standard model with massless neutrinos, we study the optical activity of a sea of neutrinos and antineutrinos. In particular, we estimate the values of the index of refraction and rotary power of this medium in the absence of dispersion.

PACS numbers: 13.15.+g, 14.60.Lm, 14.70.Bh, 95.30.Cq
\end{abstract}

\section{INTRODUCTION}

The elastic neutrino-photon scattering process, $\nu \gamma \rightarrow$ $\nu \gamma$, and the corresponding crossed channels have been the subject of numerous investigations related to astrophysical applications [1, 2, 3, 4, 5, 6, 7, 8, 9]. When the center of mass energy $\sqrt{s}$ is much less than the $W$ boson mass $m_{W}$, the amplitudes for these processes have a relatively simple form. In particular, the forward helicity non-flip amplitudes for the elastic processes $\nu \gamma \rightarrow \nu \gamma$ and $\bar{\nu} \gamma \rightarrow \bar{\nu} \gamma$ with massless neutrinos can be expanded in powers of $s / m_{W}^{2}$ by making use of unitarity 9$]$. The series are of leading order $s^{2} / m_{W}^{4}$, and the inclusion of higher powers of $s$, such as $s^{3} / m_{W}^{6}$, enables us to use the forward elastic scattering amplitudes for $\nu \gamma \rightarrow \nu \gamma$ and $\bar{\nu} \gamma \rightarrow \bar{\nu} \gamma$ to study the optical activity of a sea of neutrinos and antineutrinos. This property of a neutrino sea was recognized by Nieves and Pal [10] and studied by Mohanty, Nieves, and Pal [1] in the case where the photons move in a plasma and therefore experience dispersion. Dispersive effects give rise to mass dependent of terms of the form $k^{2} / m_{e}^{2}\left(k^{\mu}\right.$ denoting the photon momentum and $m_{e}$ the electron mass), which vanish in the vacuum. Here, we treat the vacuum case where $k^{\mu}$ satisfies $k^{2}=0$, and find a rather different dependence on $m_{W}$ and $m_{e}[12]$.

In the next section, using the results of Ref. 9], we write the forward helicity amplitudes for the elastic scattering $\nu \gamma \rightarrow \nu \gamma$ and $\bar{\nu} \gamma \rightarrow \bar{\nu} \gamma$. We then present a calculation for the index of refraction and the rotary power for a sea of neutrinos and antineutrinos as a function of the energy of an incident photon, the temperature of the sea, and the neutrino degeneracy parameter $\xi \equiv \mu / T_{\nu}$, where $\mu$ is the chemical potential for neutrinos and $T_{\nu}$ is the temperature of the sea.

\section{OPTICAL ACTIVITY}

Using the Eqs. (24)-(26) of the Ref. [9], the helicity non-flip amplitudes, $\mathcal{A}_{\lambda \lambda}^{\nu \gamma \rightarrow \nu \gamma}(s)$, for the forward elastic scattering of a photon with helicity $\lambda$ and an electron neutrino when $\sqrt{s} \ll m_{W}$, can be written as

$$
\begin{aligned}
& \mathcal{A}_{++}^{\nu \gamma \rightarrow \nu \gamma}(s)=\frac{-\alpha^{2} s^{2}}{8 m_{W}^{4} \sin ^{2} \theta_{W}} h(s), \\
& \mathcal{A}_{--}^{\nu \gamma \rightarrow \nu \gamma}(s)=\frac{-\alpha^{2} s^{2}}{8 m_{W}^{4} \sin ^{2} \theta_{W}} h(-s),
\end{aligned}
$$

where

$$
\begin{aligned}
h(s)= & -4-\frac{16}{3} \ln \left(\frac{m_{W}^{2}}{m_{e}^{2}}\right) \\
& +\frac{m_{e}^{2}}{m_{W}^{2}}\left[\left(\frac{8}{3}-\frac{8}{3} \frac{s}{m_{e}^{2}}\right) \ln \left(\frac{m_{W}^{2}}{m_{e}^{2}}\right)\right. \\
& \left.-10+\frac{64}{9} \frac{s}{m_{e}^{2}}\right] .
\end{aligned}
$$

Here, $\theta_{W}$ is the weak mixing angle, $\alpha$ is the fine structure constant, and we have neglected higher powers of $m_{e}^{2} / m_{W}^{2}$ and $s / m_{W}^{2}$. Using the Eq. (12) of the Ref. [9], the helicity non-flip amplitudes, $\mathcal{A}_{\lambda \lambda}^{\bar{\nu} \gamma \rightarrow \bar{\nu} \gamma}(s)$, for forward elastic scattering of a photon of helicity $\lambda$ and an antineutrino, can be expressed as

$$
\mathcal{A}_{\lambda \lambda}^{\bar{\nu} \gamma \rightarrow \bar{\nu} \gamma}(s)=\mathcal{A}_{-\lambda-\lambda}^{\nu \gamma \rightarrow \nu \gamma}(s)
$$

which is a consequence of $C P T$ invariance.

To calculate the optical activity of a neutrinoantineutrino sea, we consider a photon of helicity $\lambda$ and energy $\omega$ traversing a bath of neutrinos and antineutrinos that are in thermal equilibrium at the temperature $T_{\nu}$. To give an order of magnitude estimate of the index of refraction $n_{\lambda}$ of this sea, we write [13, 14]

$$
\begin{aligned}
n_{\lambda}-1= & \frac{2 \pi}{\omega^{2}} \int d N_{\nu} f_{\lambda \lambda}^{\nu \gamma \rightarrow \nu \gamma}(0) \\
& +\frac{2 \pi}{\omega^{2}} \int d N_{\bar{\nu}} f_{\lambda \lambda}^{\bar{\nu} \gamma \rightarrow \bar{\nu} \gamma}(0),
\end{aligned}
$$


which is a generalization of the Eq. (57) of the Ref. [9]. The forward-scattering amplitudes $f_{\lambda \lambda}^{\nu \gamma \rightarrow \nu \gamma}(0)$ and $f_{\lambda \lambda}^{\bar{\nu} \gamma \rightarrow \bar{\nu} \gamma}(0)$, from the Eq. (58) of the Ref. [9], are

$$
\begin{aligned}
f_{\lambda \lambda}^{\nu \gamma \rightarrow \nu \gamma}(0) & =\frac{\omega}{4 \pi s} \mathcal{A}_{\lambda \lambda}^{\nu \gamma \rightarrow \nu \gamma}(s), \\
f_{\lambda \lambda}^{\bar{\nu} \gamma \rightarrow \bar{\nu} \gamma}(0) & =\frac{\omega}{4 \pi s} \mathcal{A}_{\lambda \lambda}^{\bar{\nu} \gamma \rightarrow \bar{\nu} \gamma}(s),
\end{aligned}
$$

and $d N_{\nu}$ and $d N_{\bar{\nu}}$ are the usual Fermi-Dirac distributions,

$$
\begin{aligned}
d N_{\nu} & =\frac{1}{(2 \pi)^{3}} \frac{d^{3} \vec{p}_{\nu}}{e^{\left(E_{\nu}-\mu\right) / T_{\nu}+1}}, \\
d N_{\bar{\nu}} & =\frac{1}{(2 \pi)^{3}} \frac{d^{3} \vec{p}_{\bar{\nu}}}{e^{\left(E_{\bar{\nu}}+\mu\right) / T_{\nu}}+1} .
\end{aligned}
$$

Here, $T_{\nu}$ is the temperature of the neutrino-antineutrino sea, $\mu$ is the chemical potential for the neutrinos [15], $\vec{p}_{\nu}$ and $E_{\nu}$ are the momentum and energy of a neutrino, and $s=4 \omega E_{\nu} \sin ^{2}\left(\theta_{\nu \gamma} / 2\right)$, where $\theta_{\nu \gamma}$ is the angle between the incoming photon and the incoming neutrino (with similar definitions for the antineutrino). From Eqs. (5)-(7) we obtain

$$
\begin{aligned}
n_{\lambda}-1= & \int \frac{d N_{\nu}}{2 \omega s} \mathcal{A}_{\lambda \lambda}^{\nu \gamma \rightarrow \nu \gamma}(s) \\
& +\int \frac{d N_{\bar{\nu}}}{2 \omega s} \mathcal{A}_{\lambda \lambda}^{\bar{\nu} \gamma \rightarrow \bar{\nu} \gamma}(s) .
\end{aligned}
$$

Due to the apparent baryon asymmetry in the universe, it is natural to consider the possibility of the lepton asymmetry. To study this possibility, and for numerical calculations of the index of refraction and the rotary power, it is convenient to introduce the following ratios

$$
\begin{aligned}
L_{1} & \equiv \frac{3}{11} \frac{N_{\nu}-N_{\bar{\nu}}}{N_{\nu}+N_{\bar{\nu}}}, \\
L_{2} & \equiv \frac{2 \pi^{2}}{11 \zeta(3)} \frac{N_{\nu}-N_{\bar{\nu}}}{T_{\nu}^{3}},
\end{aligned}
$$

where $N_{\nu}$ and $N_{\bar{\nu}}$ are the number densities for the neutrino and antineutrino, respectively, and $\zeta(x)$ is the Riemann zeta function. From the Eqs. (8), (9), (11), and (12), we find

$$
\begin{aligned}
& L_{1}=\frac{1}{11} \frac{\xi^{3}+\pi^{2} \xi}{I(\xi) \xi^{3}+2 \ln 2 \xi^{2}+3 \zeta(3)}, \\
& L_{2}=\frac{1}{33 \zeta(3)}\left(\xi^{3}+\pi^{2} \xi\right),
\end{aligned}
$$

where

$$
I(\xi)=\int_{0}^{1}(1-x)^{2} \frac{e^{\xi x}-1}{e^{\xi x}+1} d x,
$$

and $\xi \equiv \mu / T_{\nu}$ is the neutrino degeneracy parameter. As it is clear from the Eqs. (13) - (15), the parameters $L_{1}$ and $L_{2}$ are solely functions of $\xi$. These neutrino asymmetry parameters $L_{1}$ and $L_{2}$ are defined such that for $|\xi| \ll 1$ they coincide with the customary definition of the neutrino asymmetry parameter $L_{\nu}$ [16] for a sea of relic neutrino-antineutrino that decoupled from photons in the early universe

$$
L_{1} \simeq L_{2} \simeq L_{\nu} \equiv \frac{N_{\nu}-N_{\bar{\nu}}}{N_{\gamma}} \simeq \frac{\pi^{2} \xi}{33 \zeta(3)} .
$$

Here, $N_{\gamma}$ is the photon number density

$$
N_{\gamma}=\frac{1}{(2 \pi)^{3}} \int \frac{2 d^{3} \vec{p}_{\gamma}}{e^{E_{\gamma} / T_{\gamma}}-1}=\frac{2 \zeta(3) T_{\gamma}^{3}}{\pi^{2}},
$$

and the relation $T_{\nu} / T_{\gamma}=(4 / 11)^{1 / 3}$, which holds for the present day temperatures of the relic neutrinos and photons, is used. When $\xi$ is large, $L_{1}$ approaches a finite value, while $L_{2}$ increases. In the Fig.1 $L_{1}$ and $L_{2}$ are plotted as functions of $\xi$. As it is clear from this figure, for $\xi \gtrsim 3, L_{1}$ is close to its maximum value of $3 / 11$.

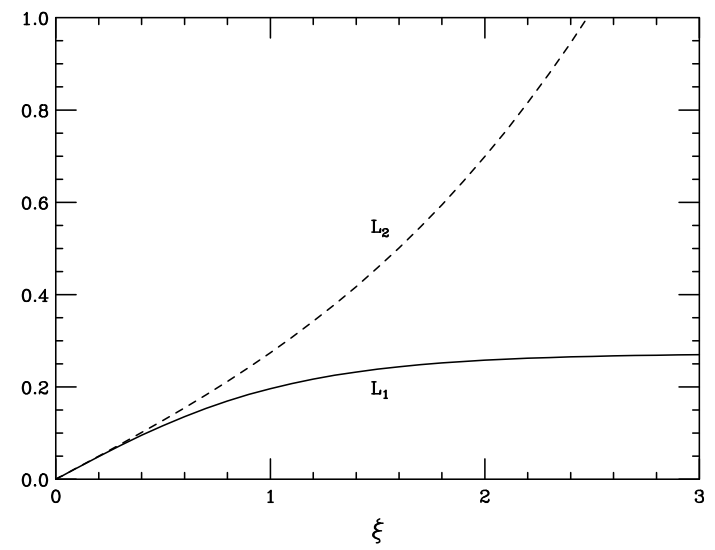

FIG. 1: The asymmetry parameters $L_{1}$ and $L_{2}$, as functions of the neutrino degeneracy parameter $\xi \equiv \mu / T_{\nu}$. The relations $L_{1}(-\xi)=-L_{1}(\xi)$ and $L_{2}(-\xi)=-L_{2}(\xi)$ hold.

After using Eqs. (8) and (9) for $d N_{\nu}$ and $d N_{\bar{\nu}}$, Eqs. (1)(41) for the amplitudes $\mathcal{A}_{\lambda \lambda}^{\nu \gamma \rightarrow \nu \gamma}(s)$ and $\mathcal{A}_{\lambda \lambda}^{\bar{\nu} \gamma \rightarrow \bar{\nu} \gamma}(s)$, and performing integrations in the Eq. (10), we obtain

$$
\begin{aligned}
& n_{+}-1=\frac{T_{\nu}^{4}}{m_{W}^{4}} c_{0}+\frac{\omega T_{\nu}^{5}}{m_{W}^{6}} c_{1}, \\
& n_{-}-1=\frac{T_{\nu}^{4}}{m_{W}^{4}} c_{0}-\frac{\omega T_{\nu}^{5}}{m_{W}^{6}} c_{1},
\end{aligned}
$$

where (for $\alpha=1 / 137$ )

$$
\begin{aligned}
c_{0}= & \frac{\alpha^{2}}{3 \pi^{2} \sin ^{2} \theta_{W}}\left[\ln \left(\frac{m_{W}^{2}}{m_{e}^{2}}\right)+\frac{3}{4}\right] \\
& \times\left(\frac{1}{4} \xi^{4}+\frac{\pi^{2}}{2} \xi^{2}+\frac{7 \pi^{4}}{60}\right) \\
\simeq & 1.9 \times 10^{-4}\left(\frac{1}{4} \xi^{4}+\frac{\pi^{2}}{2} \xi^{2}+\frac{7 \pi^{4}}{60}\right), \\
c_{1}= & \frac{4 \alpha^{2}}{9 \pi^{2} \sin ^{2} \theta_{W}}\left[\ln \left(\frac{m_{W}^{2}}{m_{e}^{2}}\right)-\frac{8}{3}\right]
\end{aligned}
$$




$$
\begin{aligned}
& \times\left(\frac{1}{5} \xi^{5}+\frac{2 \pi^{2}}{3} \xi^{3}+\frac{7 \pi^{4}}{15} \xi\right) \\
\simeq & 2.2 \times 10^{-4}\left(\frac{1}{5} \xi^{5}+\frac{2 \pi^{2}}{3} \xi^{3}+\frac{7 \pi^{4}}{15} \xi\right) .
\end{aligned}
$$

Notice that the $\xi$-dependent parts of the $L_{2}, c_{0}$, and $c_{1}$ are related as

$$
\begin{aligned}
\frac{1}{4} \frac{\partial}{\partial \xi}\left(\frac{1}{5} \xi^{5}+\frac{2 \pi^{2}}{3} \xi^{3}+\frac{7 \pi^{4}}{15} \xi\right) & =\frac{1}{4} \xi^{4}+\frac{\pi^{2}}{2} \xi^{2}+\frac{7 \pi^{4}}{60} \\
\frac{\partial}{\partial \xi}\left(\frac{1}{4} \xi^{4}+\frac{\pi^{2}}{2} \xi^{2}+\frac{7 \pi^{4}}{60}\right) & =\xi^{3}+\pi^{2} \xi
\end{aligned}
$$

In the Figs.20 the coefficients $c_{0}$ and $c_{1}$ are plotted as functions of $\xi, L_{1}$, and $L_{2}$, respectively.

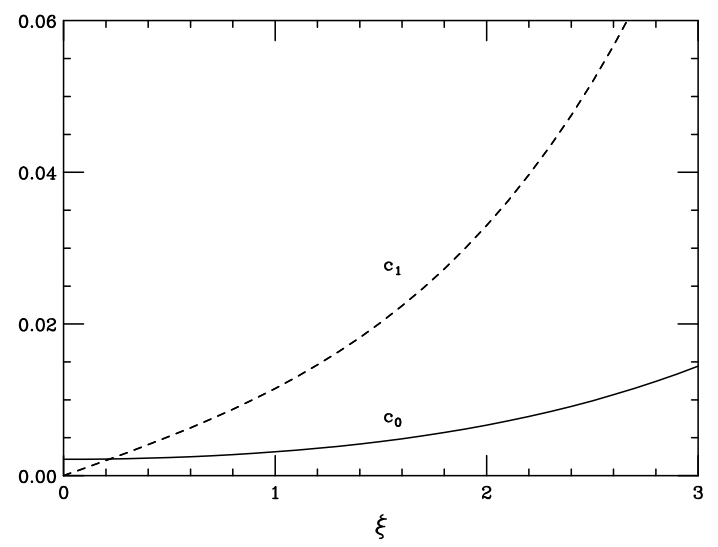

FIG. 2: The coefficients $c_{0}$ and $c_{1}$ as functions of the neutrino degeneracy parameter $\xi \equiv \mu / T_{\nu}$. The relations $c_{0}(-\xi)=$ $c_{0}(\xi)$ and $c_{1}(-\xi)=-c_{1}(\xi)$ hold.

The range of the validity of the Eqs. (18)-(21), as far as energy is concerned, is related to that of the Eqs. (11)-(3), which is $s=4 \omega E_{\nu} \sin ^{2}\left(\theta_{\nu \gamma} / 2\right) \ll m_{W}^{2}$. In the Eq. (10), if we change the upper limits of the integrations on $E_{\nu}$ and $E_{\bar{\nu}}$ from the infinity to $f T_{\nu}$, the contributions of these integrals to $c_{0}$ and $c_{1}$ in the Eqs. (18) and (19) change, at most, by $9 \%$ and $18 \%$, respectively, if we use $f=$ $\sqrt{7^{2}+\xi^{2}}$ (for $f=\sqrt{8^{2}+\xi^{2}}$, the corresponding changes are at most $5 \%$ and 10\%). Here, we set the following criterion:

$$
4 \omega f T_{\nu} \ll m_{W}^{2},
$$

which for $f=\sqrt{7^{2}+\xi^{2}}$ is

$$
\omega T_{\nu} \sqrt{7^{2}+\xi^{2}} \ll 2 \times 10^{16} \mathrm{GeV} \cdot \mathrm{K},
$$

where $\omega$ is the photon energy in $\mathrm{GeV}$, and $T_{\nu}$ is the neutrino temperature in Kelvin.

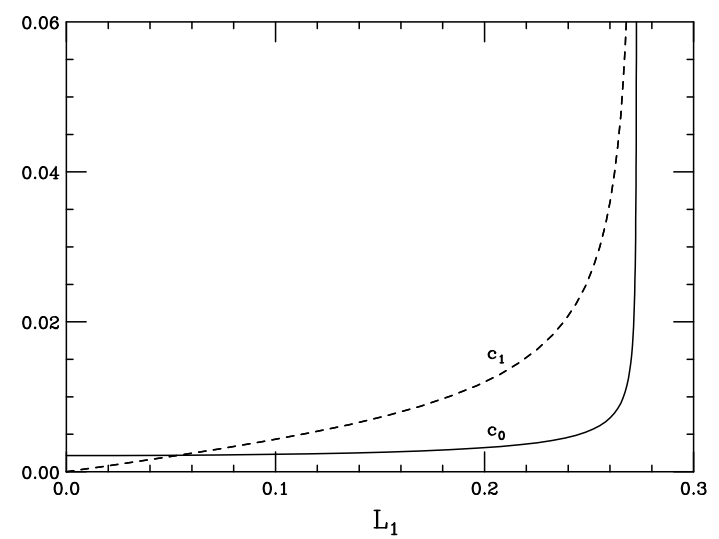

FIG. 3: The coefficients $c_{0}$ and $c_{1}$ as functions of the asymmetry parameter $L_{1}$. The relations $c_{0}\left(-L_{1}\right)=c_{0}\left(L_{1}\right)$ and $c_{1}\left(-L_{1}\right)=-c_{1}\left(L_{1}\right)$ hold.

From Eqs. (18) and (19), we have

$$
\begin{aligned}
n_{+}-n_{-} & =2 \frac{\omega T_{\nu}^{5}}{m_{W}^{6}} c_{1} \\
& \simeq 3.5 \times 10^{-77} c_{1} \omega T_{\nu}^{5},
\end{aligned}
$$

and the following approximate relation:

$$
\begin{aligned}
n_{+}-1 \simeq n_{-}-1 & \simeq \frac{T_{\nu}^{4}}{m_{W}^{4}} c_{0} \\
& \simeq 1.3 \times 10^{-60} c_{0} T_{\nu}^{4} .
\end{aligned}
$$

Equation (27) implies that the leading term of the index of refraction is independent of the helicity and the energy of the incident photon, as long as Eq. (25) is satisfied.

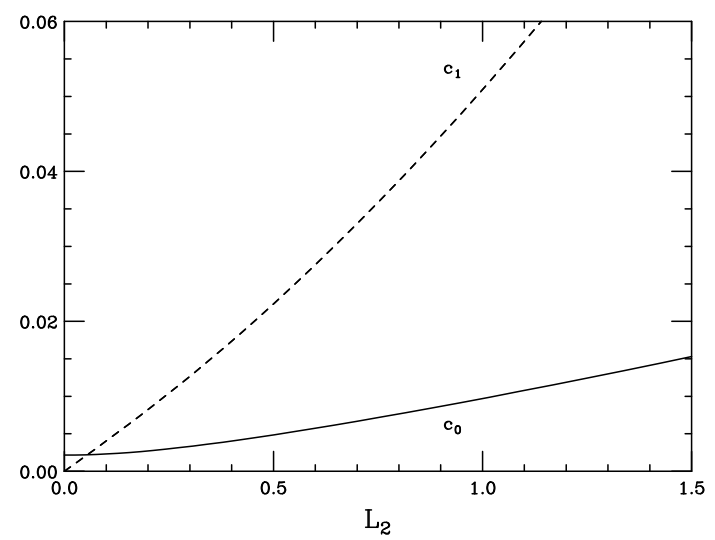

FIG. 4: The coefficients $c_{0}$ and $c_{1}$ as functions of the asymmetry parameter $L_{2}$. The relations $c_{0}\left(-L_{2}\right)=c_{0}\left(L_{2}\right)$ and $c_{1}\left(-L_{2}\right)=-c_{1}\left(L_{2}\right)$ hold.

When linearly polarized light propagates through a medium that has different indices of refraction for positive and negative helicities $\left(n_{+} \neq n_{-}\right)$, the plane of po- 
larization of the light rotates by an angle $\phi$, which is 17 .

$$
\phi=\frac{\pi}{\lambda_{\gamma}}\left(n_{+}-n_{-}\right) l=\frac{\omega}{2}\left(n_{+}-n_{-}\right) l,
$$

where $\omega$ and $\lambda_{\gamma}=2 \pi / \omega$ are the energy and wavelength of the photon and $l$ is the distance travelled by photons in the medium. To estimate the specific rotary power, $\phi / l$, for a sea of neutrinos and antineutrinos, we use Eqs. (26) and (28) to obtain

$$
\begin{aligned}
\frac{\phi}{l} & =\frac{\omega^{2} T_{\nu}^{5}}{m_{W}^{6}} c_{1} \\
& \simeq 8.9 \times 10^{-62} c_{1} \omega^{2} T_{\nu}^{5} \mathrm{rad} / \mathrm{m},
\end{aligned}
$$

where, again, $\omega$ is in $\mathrm{GeV}$ and $T_{\nu}$ is in Kelvin. A positive angle of rotation, $\phi>0$, that is $n_{+}>n_{-}$, corresponds to a clockwise rotation (dextrorotation) of the plane of polarization of the linearly polarized incident photons, as viewed by an observer that is detecting the forwardscattered light. Thus, the optical activity of a neutrinoantineutrino sea with $N_{\nu}>N_{\bar{\nu}}$ is that of a dextrorotary medium. In addition, it is clear from Eq. (29) that the rotary power, $\phi / l$, varies as $1 / \lambda_{\gamma}^{2}$, which is the same as that of quartz and most transparent substances for visible light.

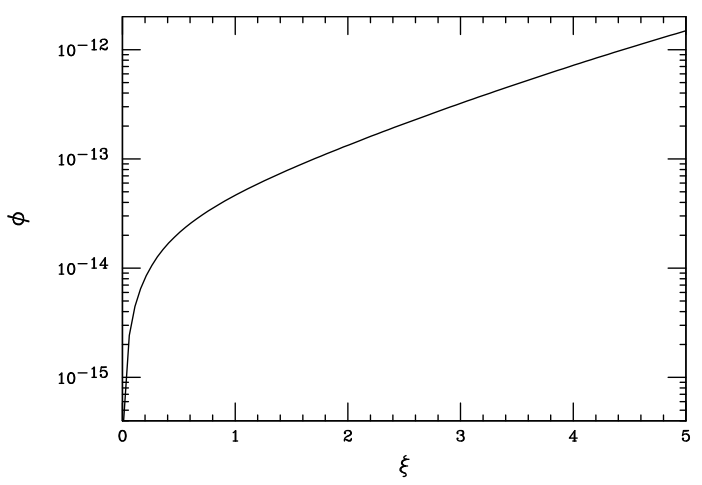

FIG. 5: The polarization rotation angle $\phi$ is shown as a function of $\xi$ for $\omega=10^{11} \mathrm{GeV}$. The curve scales as $\omega^{2}$.

To get a rough estimate of rotation angle $\phi$ for linearly polarized photons propagating through the relic neutrino and antineutrino sea (with $\xi=0.01$ ), we use Eq. (29) with $l=c t, c=3 \times 10^{8} \mathrm{~m} / \mathrm{s}, t \sim 15 \times 10^{9}$ years, $T_{\nu} \sim 2 \mathrm{~K}$, and $\omega \sim 10^{20} \mathrm{eV}$, and we find

$$
\phi \sim 4 \times 10^{-16} \mathrm{rad},
$$

which is exceedingly small. The dependence of $\phi$ on $\xi$ is shown in Fig. It should be noted that recent studies which include neutrino oscillation effects in the derivation of cosmological bounds on $\xi$ conclude that $|\xi| \lesssim 0.118$, 19, 20].

\section{CONCLUSIONS}

In conclusion, we note that using Eqs. (12), (14), (21), and (29), for the neutrino degeneracy parameter $|\xi| \ll 1$, the specific rotary power, $\phi / l$, for a sea of neutrinos and antineutrinos is

$$
\frac{\phi}{l}=\frac{112 \pi G_{F} \alpha}{45 \sqrt{2}}\left[\ln \left(\frac{m_{W}^{2}}{m_{e}^{2}}\right)-\frac{8}{3}\right] \frac{\omega^{2} T_{\nu}^{2}}{m_{W}^{4}}\left(N_{\nu}-N_{\bar{\nu}}\right) .
$$

This equation differs in several respects from the corresponding result in Ref. [1]],

$$
\frac{\phi}{l}=\frac{G_{F} \alpha}{3 \sqrt{2} \pi}\left(N_{\nu}-N_{\bar{\nu}}\right) \frac{\omega_{P}^{2}}{m_{e}^{2}},
$$

where, in the non-relativistic limit, the plasma frequency $\omega_{P}$ is related to the electron number density $n_{e}$ as,

$$
\omega_{P}^{2}=\frac{4 \pi \alpha n_{e}}{m_{e}} .
$$

From Eqs. (31) and (32), it is clear that the mass scales for the case of photon dispersion versus no dispersion are very different and this favors the dispersive regime. The size of specific rotary power in the dispersive case is independent of the frequency of the propagating photons and, apart from a dependence on the lepton asymmetry parameter $L_{\nu} \propto N_{\nu}-N_{\bar{\nu}}$ shared by both expressions, is controlled by the electron number density $n_{e}$. In addition, the non-dispersive case depends on frequency and temperature. These differences clearly indicate that Eqs. (29) and (32) represent complementary limits in the treatment of the optical activity of a neutrino-antineutrino sea.

\section{Acknowledgments}

We wish to thank Duane Dicus for numerous helpful discussions. One of us (A.A.) wishes to thank the Department of Physics and Astronomy at Michigan State University for its hospitality and computer resources. This work was supported in part by the National Science Foundation under Grant No. PHY-0070443.

2, 359 (1971)

[1] H.-Y. Chiu and P. Morrison, Phys. Rev. Lett. 5, 573 (1960).

[2] M.J. Levine, Nuovo Cimento A 48, 67 (1967).

[3] L.F. Landovitz and W.M. Schreiber, Nuovo Cimento A
[4] V.K. Cung and M. Yoshimura, Nuovo Cimento A 29, 557 (1975).

[5] D.A. Dicus and W.W. Repko, Phys. Rev. D 48, 5106 
(1993).

[6] J. Liu, Phys. Rev. D 44, 2879 (1991).

[7] A. Abbasabadi, A. Devoto, D.A. Dicus, and W.W. Repko, Phys. Rev. D 59, 013012 (1999).

[8] A. Abbasabadi, A. Devoto, and W.W. Repko, Phys. Rev. D 63, 093001 (2001).

[9] A. Abbasabadi and W.W. Repko, Phys. Rev. D 64, 113007 (2001).

[10] J.F. Nieves and P.B. Pal, Phys. Rev. D 39, 652 (1989).

[11] S. Mohanty, J.F. Nieves and P.B. Pal, Phys. Rev. D 58, 093007 (1998).

[12] G. Karl and V. Novikov, "Optical activity of a neutrino sea in the Standard Model," hep-ph/0009012

[13] Notice that Eq. (5) is based on the standard Lorentz relation that connects the index of refraction to the forwardscattering amplitude, for a medium that is at $T=0$ temperature. Here, we make a nontrivial assumption that the introduction of finite temperature in the medium, will not spoil the coherent condition that was necessary to derive the Lorentz relation. For a similar approach, see P. Langacker and J. Liu, Phys. Rev. D 46, 4140 (1992).

[14] M.L. Goldberger and K.M. Watson, Collision Theory
(Wiley, New York, 1964), p. 771.

[15] P.J.E. Peebles, Principles of Physical Cosmology (Princeton University Press, Princeton, NJ, 1993), p. 160.

[16] J. Lesgourgues and S. Pastor, Phys. Rev. D 60, 103521 (1999).

[17] For a discussion of the rotation of the polarization plane of a linearly polarized light in a gas, see the discussion leading to the Eq. (8) of V.G. Baryshevsky, "Timereversal-violating birefringence of photon in a medium exposed to electric and magnetic field," hep-ph/0007353. For a general discussion of rotary power, see J.P. Mathieu, Optics (Pergamon Press, New York, 1975), p. 235; M.V. Klein, Optics (Wiley, New York, 1970), p. 502.

[18] A. D. Dolgov, S. H. Hansen, S. Pastor, S. T. Petcov, G. G. Raffelt and D. V. Semikoz, Nucl. Phys. B 632, 363 (2002) hep-ph/0201287

[19] K. N. Abazajian, J. F. Beacom and N. F. Bell, Phys. Rev. D 66, 013008 (2002) astro-ph/0203442

[20] Y. Y. Wong, Phys. Rev. D 66, 025015 (2002) hep-ph/0203180 